\title{
Genotipificación de Mycobacterium leprae Colombiano para la Determinación de Patrones de Transmisión de la Enfermedad
}

\author{
Genotyping Colombian Mycobacterium leprae for determining \\ disease transmission patterns
}

\author{
José F. Torres-Ávila, Claudia L. Colorado, Luís A. Gamboa, María J. Araujo, \\ Clara I. León-Franco y Martha I. Guerrero-Guerrero
}

Centro Dermatologico Federico Lleras Acosta. Bogotá, Colombia. fernandot85@gmail.com claucolor@yahoo.com,gamboaluisart@yahoo.com,mariaaraujo@yahoo.com, clarainesleon@yahoo.com, marthainiridag@yahoo.com,

Recibido 4 Agosto 2008/Enviado para Modificación 5 Enero 2009/Aceptado 15 Enero 2009

\section{RESUMEN}

Objetivo Evaluar la variabilidad de VNTR (variable-number tandem repeat) de Mycobacterium leprae de pacientes colombianos con y sin tratamiento previo para identificar posibles fuentes de infección y entender los patrones de transmisión de la enfermedad.

Metodología Estudio transversal descriptivo, en donde mediante un muestreo electivo a conveniencia se tomaron 161 biopsias de pacientes multibacilares de lepra, que habían sido solicitadas para diagnóstico y seguimiento de la enfermedad, de las cuales se realizó extracción de ADN de $M$. leprae y usando la técnica de PCR para VNTRs de $M$. leprae estandarizada, se establecieron los genotipos y los diferentes clusters mediante el agrupamiento apareado UPGMA.

Resultados En las 161 muestras totales se hallaron 22 genotipos VNTRs diferentes, de las cuales 100 muestras (62,1\%) pertenecían al genotipo único VNTRU, y de los genotipos restantes, los mayoritarios, es decir los que dieron lugar a formación de grupos o clusters fueron VNTR17 (5,6\%), VNTR20 (4,3\%), VNTR18 (4,3\%), VNTR14 (4,3\%) y VNTR13 (3,7\%).

Conclusión En este estudio se evidencia por análisis de agrupamiento que se pueden detectar clones con diferente grado de virulencia/agresividad, lo cual implica la necesidad de incrementar varias de las actividades del programa de control que darán como resultado la verdadera disminución de la transmisión del microorganismo.

Palabras Clave: Mycobacterium leprae, lepra, epidemiología molecular, dermatoglifia del ADN, secuencias repetidas en tandem, Colombia (fuente: DeCS, BIREME). 


\begin{abstract}
Objective Assessing VNTR (variable-number tandem repeat) variability of Mycobacterium leprae from Colombian patients with and without prior treatment to identify potential sources of infection and to understand the patterns of disease transmission.

Methodology This was a descriptive cross-sectional study where a convenience sample of biopsies was taken from 161 multibacillary leprosy patients; diagnosis and monitoring of the disease had been requested for these patients. DNA was extracted from $M$. leprae and standardised using the PCR technique for M. leprae VNTR, ge-notypes were established and different clusters grouped by unweighted pair group method with arithmetic mean (UPGMA).

Results 22 different VNTR genotypes were found from 161 samples, of which 100 samples $(62.1 \%)$ had a single $u$-VNTR genotype and the remaining genotypes were VNTR 17 (5.6\%), VNTR 20 (4.3\%), VNTR 18 (4.3\%), VNTR 14 (4.3\%) and VNTR $13(3.7 \%)$, namely those forming groups or clusters.

Conclusion This study showed that clones can be detected with varying degrees of virulence / aggressiveness by cluster analysis, implying the need for more monitoring programme activities which will result in a real decline in microorganism transmission.
\end{abstract}

Key Words: Mycobacterium leprae, leprosy, epidemiology, molecular, DNA finger-printing, tandem repeat sequence, Colombia (source: MeSH, NLM).

1 ecientemente la OMS ha reportado que la carga de lepra se ha reducido, pero que en el futuro van a seguir apareciendo nuevos casos (1). En el caso de Colombia actualmente se reporta una lenta tendencia a la disminución de la incidencia de lepra debido a la baja en los reportes de un año a otro (2005 a 2006). Esta disminución de casos, probablemente no sea real sino que la búsqueda de casos actualmente puede ser menor (1).

Estudios realizados acerca de la transmisión y la identificación de las fuentes de infección de lepra, han sido difíciles de realizar debido al largo período de incubación de la enfermedad y aparición de las manifestaciones clínicas, y a que el $M$. leprae sigue siendo un microorganismo no cultivable en medios artificiales (2). Todos estos factores, han influido en retardar el entendimiento de las rutas de transmisión de $M$. leprae y se siguen haciendo esfuerzos en el diseño de estrategias efectivas en el enfoque quimioterapéutico para la eliminación de la enfermedad.

Por tanto, los métodos de tipificación de cepas de M. leprae pueden ser de gran ayuda para el conocimiento epidemiológico de este microorganismo y ayudar a identificar las fuentes de infección para entender sus patrones de transmisión y poder diferenciar entre los eventos de recaída y reinfección (3). 
Hoy en día la transmisión de este microorganismo se ha abordado con el enfoque de la epidemiología molecular, y esta, podría darnos claras explicaciones, debido a que con estas metodologías se usan marcadores genéticos del microorganismo como son las secuencias repetitivas y los polimorfismos VNTR (Variable-Number Tandem Repeat) y más recientemente la genotipificación de Mycobacterium leprae mediante el uso de micro satélites que son unidades repetitivas de 6 a 100pb que son excelentes fuentes de polimorfismo, que nos sirven para investigar la diversidad genética en una población bacterial y además resulta ser un instrumento útil para rastrear cadenas de transmisión cortas (4).

Varios estudios aplicando técnicas moleculares como son el análisis de RFLP de diferentes secuencias blanco incluyendo las repeticiones MLEP, polimorfismos conformacionales de cadena simple y los polimorfismos en la secuencia del ADN de la región interna de la trascripción (ITS entre 16S y 23S de rRNA), han sido llevados a cabo, pero no han evidenciado asociación con variabilidad de los aislamientos (4). Además, ha sido descrita una es-tructura única polimórfica del gen polA y de 3 a 4 copias repetidas de GA-CATC en el gen rpoT pero su valor para identificar genotipos de $M$. leprae ha sido limitada (3). Recientes desarrollos en tipificación molecular y de análisis de genomas in silico han revelado que el análisis del número de secuencias VNTRs podría ser una herramienta promisoria para la tipificación de cepas. En un reporte reciente se observó variación en 10 de 37 copias de un único locus de repetición TTC en $M$. leprae sugiriendo aplicabilidad de esta genotipificación para estudios de epidemiología molecular (5).

Usando MIRUs (Mycobacterial Interspersed Repetitive Unit) así como un blanco VNTR para tipificación, en $M$. tuberculosis, se ha comprobado que es necesario realizar análisis simultáneos de repeticiones en diferentes loci para obtener suficiente poder de diferenciación entre cepas, lo cual se ha extrapolado al estudio de M. leprae (6).

Análisis in silico del genoma de M. leprae identificaron algunas de las secuencias VNTRs en el genoma y desarrollando 4 sistemas individuales de PCR, demostraron por electroforesis en gel de agarosa y por secuenciación, la asociación entre el tamaño de las secuencias y la variabilidad de la cepa de $M$. leprae de los pacientes de Rió de Janeiro (7). La variabilidad del tamaño de los VNTRs observada en dichos pacientes fue confirmada en pacientes de diferentes partes del mundo, luego ellos mismos demostraron la especificidad de estos sistemas de PCR para M. leprae aplicando este sistema a varias especies de micobacterias y a ADN humano (7). 
Adicionalmente se obtuvo exitosamente productos de amplificación de los 4 sistemas desarrollados, a partir de muestras de biopsias congeladas de pacientes multibacilares y paucibacilares, de muestras de linfa tanto de pacientes multibacilares y paucibacilares y de algunas biopsias de nervio de pacientes con lepra neural pura. Posteriormente se dieron cuenta que todos los sistemas permitieron obtener productos de PCR a partir de muestras de biopsia embebidas en parafina permitiendo así realizar estudios retrospectivos y evaluar modificación o migración de cepas de $M$. leprae a través de periodos largos de tiempo (3).

En este estudio se propuso por lo tanto, evaluar la variabilidad de VNTR de Mycobacterium leprae de pacientes colombianos con y sin tratamiento previo para identificar posibles fuentes de infección y entender los patrones de transmisión de la enfermedad.

\section{MÉTODOS}

Se realizó un estudio de corte transversal descriptivo, incluyendo biopsias de piel de pacientes colombianos que asistieron al Centro Dermatológico Federico Lleras Acosta E.S.E. (CDFLLA) durante el periodo 2000 a 2004, en busca de diagnóstico o seguimiento del tratamiento de lepra, usando un muestreo electivo a conveniencia, en el cual se encontraron en el banco de biopsias institucional 435 biopsias correspondientes a pacientes atendidos durante este periodo, de las cuales se tomaron 161 biopsias que cumplían con los requerimientos para su estudio y análisis; correspondiendo 85 biopsias a las que se tomaron para hacer seguimiento o control de la enfermedad de Hansen y 79 biopsias para realizar diagnóstico.

Obtención de las muestras de ADN

Se realizaron 6 cortes de $4 \mu \mathrm{m}$ a cada muestra de biopsia incluida en parafina, con un micrótomo Leyca ${ }^{\circledR}$, se almacenaron a temperatura ambiente hasta su procesamiento y a partir de estas muestras se realizó extracción de ADN total con el método de Triton- X100 al $1 \%$. La calidad del ADN extraído se evaluó mediante una corrida electroforética de 5 ì en gel de agarosa al 0,8\%.

Selección de los iniciadores para amplificación

Después de revisar la literatura disponible, así como las bases de datos Le-proma (8), Lgmb (9) y Sanger (10), se escogieron entre las secuencias más usadas en la tipificación de cepas de $M$. leprae, las que encontramos más relevantes que fueron las siguientes: GAA, AT17, TA18, GTA9 $(3,4)$, amablemente donados por Patrick Brennan, de la Universidad de Colorado, USA. 
Estandarización

Para realizar la genotipificación de $M$. leprae con los marcadores seleccionados (GAA, AT17, TA18, GTA9) se estandarizó y optimizó previamente cada parámetro como fue las concentraciones de $\mathrm{MgCl}_{2}, \mathrm{dNTP}$ 's, iniciadores para los 4 blancos, y del ADN plantilla; igualmente la temperatura de anillamiento y el número de ciclos. Como controles se usaron tres muestras de ADN de $M$. leprae de referencia donado por la Universidad de Colorado (USA), que fueron T-53, TM-4923 y 4316.

Amplificación

Una vez optimizados los parámetros para cada uno de los cuatro blancos moleculares se realizó amplificación de todas las muestras usando las condi-ciones óptimas en cada caso, para un volumen final de $25 \mu$ l.

Obtención de patrones genotípicos

Los productos de PCR de cada uno de los cuatro VNTRs, fueron usados para realizar corridos electroforéticos, empleando $10 \mu \mathrm{l}$ del producto de PCR de cada blanco molecular en un gel de agarosa del 2,5 \% (Invitrogen). Como marcador de peso molecular se uso un ADN leader $100 \mathrm{pb}$ (Sigma), entre 100 y $1000 \mathrm{pb}$ en los dos extremos del gel. Los geles fueron fotografiados y posteriormente se obtuvo archivos digitales de ellos.

Análisis

Se realizó un análisis de agrupamientos, a partir de una matriz de similaridad construyendo los respectivos dendogramas, previa inclusión y homogenización de los archivos digitalizados, en el programa de análisis GelComparII (www.appliedMath's.com) cuyo acceso fue gentilmente proporcionado por el fabricante.

El análisis o comparación de los patrones de bandas (Fingerprint) se hizo basado en la posición combinada de las cuatro bandas, utilizando el coefi-ciente Dice que dio lugar a la matriz de similaridad. A partir de la matriz se realizó un análisis de agrupamientos basado en UPGMA y el dendograma se obtuvo optimizando al $1 \%$ y con una tolerancia del $1 \%$ en la posición de las bandas y se le dio igual peso a cada uno de los cuatro sistemas VNTRs usado. Teniendo como base los grupos o clusters obtenidos, se determino las proporciones de cada una de las variables demográficas, clínicas y epidemiológicas que ellos contenían y se compararon entre sí con el fin de identificar los agrupamientos o clones con características específicas. 


\section{RESULTADOS}

En la población estudiada se encontraron 22 genotipos diferentes y la mayor proporción de estos genotipos correspondió al 62,1\% de las muestras con genotipo único (VNTRs U).

Tabla 1. Descripción de las variables demográficas en relación con los genotipos

\begin{tabular}{|c|c|c|c|c|c|c|}
\hline \multirow{2}{*}{$\begin{array}{c}\text { Variables } \\
\text { Demográficas }\end{array}$} & \multicolumn{6}{|c|}{ Genotipos } \\
\hline & $\begin{array}{c}\text { VNTRs U } \\
\mathrm{N}(\%)\end{array}$ & $\begin{array}{l}\text { VNTRs17 } \\
N(\%)\end{array}$ & $\begin{array}{l}\text { VNTRs20 } \\
\mathrm{N}(\%)\end{array}$ & $\begin{array}{c}\text { VNTRs18 } \\
N(\%)\end{array}$ & $\begin{array}{l}\text { VNTRs14 } \\
\mathrm{N}(\%)\end{array}$ & $\begin{array}{c}\text { VNTRs13 } \\
\mathrm{N}(\%)\end{array}$ \\
\hline \multicolumn{7}{|l|}{ Género } \\
\hline $\begin{array}{l}\text { Masculino } \\
\text { Femenino }\end{array}$ & $\begin{array}{l}75(75) \\
25(25)\end{array}$ & $\begin{array}{l}8(88,9) \\
1(11,1)\end{array}$ & $7(100)$ & $7(100)$ & $7(100)$ & $\begin{array}{l}4(66,7) \\
2(33,3)\end{array}$ \\
\hline \multicolumn{7}{|c|}{ Edad en el momento del diagnostico } \\
\hline$>1-10$ años & $3(3)$ & & & & & \\
\hline$>11-20$ años & $7(7)$ & $2(22,2)$ & & & $1(14,3)$ & \\
\hline$>21-30$ años & $21(21)$ & $3(33,3)$ & $2(28,6)$ & $1(14,3)$ & $3(42,9)$ & $1(16,6)$ \\
\hline$>31-40$ años & $24(24)$ & & $1(14,3)$ & $3(42,9)$ & & $4(66,7)$ \\
\hline$>41-50$ años & $19(19)$ & $2(22,2)$ & & $2(28,6)$ & $1(14,3)$ & \\
\hline$>51-60$ años & $14(14)$ & $1(11,1)$ & $1(14,3)$ & $1(14,3)$ & $1(14,3)$ & \\
\hline$>61-70$ años & $10(10)$ & $1(11,1)$ & & & & $1(16,6)$ \\
\hline$>71-80$ años & $2(2)$ & & $3(42,9)$ & & $1(14,3)$ & \\
\hline \multicolumn{7}{|c|}{ Departamento de nacimiento } \\
\hline Santander & $23(23,7)$ & $2(22,2)$ & $3(42,9)$ & $1(14,3)$ & $4(57,1)$ & $1(16,6)$ \\
\hline Tolima & $21(21,6)$ & $1(11,1)$ & $2(28,6)$ & & $1(14,3)$ & $1(16,6)$ \\
\hline Cundinamarca & $16(16,5)$ & & $1(14,3)$ & $1(14,3)$ & $1(14,3)$ & $1(16,6)$ \\
\hline Boyacá & $16(16,5)$ & $1(11,1)$ & & $3(42,9)$ & & $2(33,3)$ \\
\hline Otros & $24(21.7)$ & $5(55,5)$ & $1(14,3)$ & $2(28,6)$ & $1(14,3)$ & $1(16,6)$ \\
\hline \multicolumn{7}{|c|}{ Municipio de nacimiento } \\
\hline Suaita & $6(6)$ & & & & & $1(16,6)$ \\
\hline Bolivar & $5(5)$ & & & & & \\
\hline Ibagué & 4(4) & & & & & \\
\hline Otros & $85(85)$ & $9(100)$ & $7(100)$ & $7(100)$ & $7(100)$ & $5(83,4)$ \\
\hline \multicolumn{7}{|c|}{ Departamento de procedencia } \\
\hline Cundinamarca & $79(79)$ & $5(55,5)$ & $6(85,7)$ & $6(85,7)$ & $4(57,1)$ & $4(66,6)$ \\
\hline Otros & $21(21)$ & $4(44,5)$ & $1(14,3)$ & $1(14,3)$ & $3(42,9)$ & $2(33,3)$ \\
\hline \multicolumn{7}{|c|}{ Municipio de procedencia } \\
\hline Bogotá & $61(61)$ & $4(4$ & $6(85,7)$ & $5(71$ & ,1) & $4(66,6)$ \\
\hline Otros & $39(39)$ & $5(55,5)$ & $1(14,3)$ & $2(28,6)$ & $3(42,9)$ & $2(33,3)$ \\
\hline Totales & $100(62,1)$ & $9(5,6)$ & $7(4,3)$ & $7(4,3)$ & $7(4,3)$ & $6(3,7)$ \\
\hline
\end{tabular}

Entre los genotipos restantes, los mayoritarios, es decir los que dieron lugar a formación de grupos o clusters fueron VNTR17 9(5,6 \%), VNTR20 7(4,3 $\%)$, VNTR18 7(4,3\%), VNTR14 7(4,3\%), y VNTR13 6(3,7 \%).

Interesante resaltar que se encontraron miniclusters, es decir agrupamientos de 3 ó 4 cepas que fueron los genotipos VNTR 11, VNTR 12, VNTR15 y VNTR9. Los principales hallazgos obtenidos del análisis de las variables demográficas, clínicas y epidemiológicas en relación con los genotipos encontrados, se presentan en las Tablas 1,2 y 3 respectivamente. 
Tabla 2. Descripción de las variables clínicas en relación con los genotipos VNTRs mayoritarios encontrados

\begin{tabular}{|c|c|c|c|c|c|c|}
\hline \multirow{2}{*}{ Variables Clínicas } & \multicolumn{6}{|c|}{ GENOTIPOS } \\
\hline & $\begin{array}{c}\text { VNTRU } \\
\mathrm{N}(\%)\end{array}$ & $\begin{array}{c}\text { VNTR17 } \\
\mathrm{N}(\%)\end{array}$ & $\begin{array}{c}\text { VNTR20 } \\
N(\%)\end{array}$ & $\begin{array}{c}\text { VNTR18 } \\
\mathrm{N}(\%)\end{array}$ & $\begin{array}{c}\text { VNTR14 } \\
\mathrm{N}(\%)\end{array}$ & $\begin{array}{c}\text { VNTR13 } \\
\mathrm{N}(\%) \\
\end{array}$ \\
\hline \multicolumn{7}{|l|}{ Clașificación clínica } \\
\hline LL & $69(69)$ & $5(55,6)$ & $7(100)$ & $5(71,4)$ & $5(71,4)$ & $4(66,6)$ \\
\hline LNP & 12(12) & $3(33,3)$ & & $1(14,3)$ & $2(28,6)$ & $2(33,3)$ \\
\hline Otro & $19(19)$ & $1(11,1)$ & & $1(14,3)$ & & \\
\hline \multicolumn{7}{|c|}{ Momento de la toma de la biopsia } \\
\hline Diagnóstico & $33(33)$ & $8(88,9)$ & $3(42,9)$ & $6(85,7)$ & $7(100)$ & $2(33,3)$ \\
\hline Sospecha & $46(46)$ & $1(11,1)$ & $3(42,9)$ & $1(14,3)$ & & $1(16,6)$ \\
\hline \multicolumn{7}{|c|}{ Resistencia/Persistencia/Recaída } \\
\hline Otro & $21(21)$ & & $1(14,2)$ & & & $3(50,1)$ \\
\hline \multicolumn{7}{|l|}{ Zn biopsia } \\
\hline Multibacilar & $66(66)$ & $9(100)$ & $7(100)$ & $7(100)$ & $7(100)$ & $4(66,6)$ \\
\hline Paucibacilar & $34(34)$ & & & & & $2(33,3)$ \\
\hline \multicolumn{7}{|l|}{ Tratamiento previo } \\
\hline Con TTO previo & $67(67)$ & $1(11,1)$ & $4(57,1)$ & $1(14,3)$ & & $4(66,6)$ \\
\hline Sin TTO previo & $33(33)$ & $8(88,9)$ & $3(42,9)$ & $6(85,7)$ & $7(100)$ & $2(33,3)$ \\
\hline \multicolumn{7}{|l|}{ Con resistencia a } \\
\hline Dapsona (DDS) & $3(3)$ & & & & & \\
\hline Rifampicina & $3(3)$ & & $1(14,3)$ & & $3(42,9)$ & $1(16,6)$ \\
\hline Ofloxacina & $4(4)$ & & & & & \\
\hline Sin Resistencia & $90(90)$ & $9(100)$ & $6(85,7)$ & $7(100)$ & $4(57,1)$ & $5(83,4)$ \\
\hline \multicolumn{7}{|c|}{ Regularidad del tto previo } \\
\hline Regular & $51(51)$ & & $2(28,6)$ & & & $2(33,3)$ \\
\hline Irregular & $9(9)$ & & $2(28,6)$ & & & \\
\hline Otro & $40(40)$ & $9(100)$ & $3(42,9)$ & $7(100)$ & $7(100)$ & $4(66,6)$ \\
\hline Totales & $100(62,1)$ & $9(5,6)$ & $7(4,3)$ & $7(4,3)$ & $7(4,3)$ & $6(3,7)$ \\
\hline
\end{tabular}

\section{DISCUSIÓN}

La genotipificación del M. leprae en este estudio se abordó pensando que podría ser de gran valor para el conocimiento epidemiológico de este microorganismo y ayudarnos a identificar las fuentes de infección, permitiendo entender sus patrones de transmisión, y distinguir entre los eventos de recaída y reinfección.

Recientes desarrollos en tipificación molecular y de análisis de genomas in silico revelaron que el análisis del número de secuencias VNTRs son una herramienta promisoria para la tipificación de cepas.

Aunque muy recientemente el grupo de Cole S, publica un estudio en el que aseguran que estos 4 VNTRs no son lo suficientemente estables en el tiempo como para ser usados como marcadores moleculares robustos, sin embargo, existen varios estudios como los arriba mencionados, que evidencian lo contrario (11). 
Tabla 3. Descripción de las variables epidemiológicas en relación con los genotipos VNTRs mayoritarios encontrados

\begin{tabular}{|c|c|c|c|c|c|c|}
\hline \multirow{2}{*}{$\begin{array}{c}\text { Variables } \\
\text { epidemiológicas }\end{array}$} & \multicolumn{6}{|c|}{ Genotipos } \\
\hline & $\begin{array}{l}\text { VNTRU } \\
\text { N (\%) }\end{array}$ & $\begin{array}{l}\text { VNTR17 } \\
\mathrm{N}(\%)\end{array}$ & $\begin{array}{c}\text { VNTR20 } \\
\mathrm{N}(\%)\end{array}$ & $\begin{array}{c}\text { VNTR18 } \\
\mathrm{N}(\%) \\
\end{array}$ & $\begin{array}{c}\text { VNTR14 } \\
\text { N }(\%) \\
\end{array}$ & $\begin{array}{c}\text { VNTR13 } \\
\text { N }(\%) \\
\end{array}$ \\
\hline \multicolumn{7}{|l|}{ Posible caso índice } \\
\hline 1er Grado & $21(21)$ & & & $1(14,3)$ & $1(14,3)$ & \\
\hline 2do Grado & $9(9$ & $2(22,2)$ & & & & $1(1$ \\
\hline Otro & $70(7$ & & $7(100)$ & $6(85,7)$ & $3(4$ & \\
\hline \multicolumn{7}{|l|}{ Clasificación OMS } \\
\hline $\begin{array}{l}\text { Multibacilar } \\
\text { Paucibacilar }\end{array}$ & $89(89)$ & $9(100)$ & $6(85,7)$ & $7(100)$ & $7(100)$ & $6(100)$ \\
\hline \multicolumn{7}{|c|}{ Tiempo de evolucićn de la enfermedad(meses) } \\
\hline $\begin{array}{l}>1-100 \\
>101-200\end{array}$ & $72(72)$ & $5(55,6)$ & $6(85,7)$ & $7(100)$ & $5(71,4)$ & $\begin{array}{l}1(16,6) \\
2(33,2)\end{array}$ \\
\hline $\begin{array}{r}1-200 \\
\text { Otro }\end{array}$ & $\begin{array}{l}18(18) \\
10(10)\end{array}$ & $\begin{array}{l}1(11,1) \\
3(33,3)\end{array}$ & ,3) & & $2(28,6)$ & $\begin{array}{l}2(33,2) \\
3(49,8)\end{array}$ \\
\hline Totales & $100(62,1)$ & $9(5,6)$ & $7(4,3)$ & $7(4,3)$ & $7(4,3)$ & $6(3,7)$ \\
\hline
\end{tabular}

Entre los 22 genotipos encontrados, con el sistema estandarizado y optimizado para este estudio, tres de los genotipos mayoritarios agruparon 100\% de individuos de genero masculino, lo cual podría indicar predilección por este tipo de hospederos, sugiriendo que es un marcador de cepas poco virulentas que prefieren el hospedero menos resistente que les permita multiplicarse mas fácilmente, característica que se refuerza cuando observamos que estos genotipos también son mas comunes entre los individuos pertenecientes a grupos de edad mayores que los promedios encontrados en este estudio (21-41 años) y que los reportados como los de mayores incidencias en Colombia (1544 años) como en el caso especifico del genotipo VNTR20. Igualmente, este sería un hallazgo que nos explicaría el hecho de que se encuentren tiempos de incubación de la enfermedad tan amplios como los reportados en la epidemiologia clásica de la lepra de 2 a 20 años (12), implicando la existencia de diferentes clones de $M$. leprae con diferentes grados de virulencia, como se ha reportado en otras bacterias (13). En consecuencia, se evidencia que si aun circulan genotipos poco virulentos en la comunidad, es porque las medidas de control no han sido lo suficientemente efectivas como para eliminarlos y que la enfermedad se está perpetuando a costa de estos casos que se demoran hasta 20 años en evidenciarse clínicamente, dando la impresión de que tal comunidad se encuentra en etapa de pos eliminación de la enfermedad como actualmente se cree en Colombia.

En el ámbito mundial mucho se ha discutido sobre la existencia de susceptibilidad genética para desarrollar lepra, por el hecho de existir familias de leprosos como existe de tuberculosos, en los cuales el gen NRAMP está implicado (2), y esto es ahora un paradigma ampliamente aceptado para la mayoría 
de las enfermedades infecciosas (14); específicamente en lepra, se han identificado genes en la región HLA como factores de susceptibilidad del hospedero para la lepra, también se han identificado locus de susceptibilidad en la región cromosomal 10p13 en familias de la India (15) y en Brasil el papel que tendría la región $6 p 21$ y $17 q 22$ en esta susceptibilidad (16); por lo tanto, nuestro hallazgo sobre la mayor frecuencia de los genotipos VNTRU, VNTR20, VNTR17 y VNTR14 en los enfermos oriundos de Santander, y de los genotipos VNTR18 y VNTR13 en los nacidos en Boyacá, es totalmente concordante con tales aseveraciones, teniendo en cuenta que la presencia de factores genéticos del hospedero incrementa el riesgo de sufrir la enfermedad después de la exposición al agente infeccioso. Este hallazgo implica que desde la perspectiva de la salud pública, se deben incrementar medidas de control tan simples como son el seguimiento periódico de los convivientes para la detección precoz de la enfermedad y la rápida implementación de la quimioterapia que corte la cadena de transmisión.

En este mismo sentido encontramos que la mayor frecuencia de posible caso índice, correspondió al primero y segundo grado de parentesco, pero creemos que este evento se encuentra mas relacionado con la convivencia mas estrecha que se tiene con padres y hermanos similar a lo que se ha encontrado en tuberculosis, donde se observa que se llegan a compartir no solo la casa sino también la habitación e incluso el lecho (Comunicación personal: Arbeláez MP. Universidad Antioquia; 2007).

Por ende, el encontrar genotipos con una distribución geográfica específica sería lógico desde la perspectiva del agrupamiento familiar que han reportado estudios de la epidemiología clásica de la lepra (9)

El haber encontrado varios genotipos VNTR17, VNTR18, VNTR13 y VNTR14, que agrupan exclusivamente casos de la forma LL polar, y que posee mas del $70 \%$ de muestras $\mathrm{ZN}(+)$, nos indicaría que nuestro sistema podría ser marcador de clones que se multiplican mas rápidamente que otros, indicando también que en Mycobacterium leprae existen clones más exitosos que otros como ya se ha documentado en otras bacterias (17); sin embargo, este hallazgo podría estar más relacionado con las características genéticas de susceptibilidad del hospedero, ya mencionadas, para la presentación de específicas formas clínicas de la enfermedad.

Uno de los genotipos mayoritarios mostró que es el más frecuente entre los casos ya tratados: sospechosos de resistencia/persistencia y recaídas y que el 
$50 \%$ de las cepas que agrupa, estaban causando recaídas de la enfermedad, hallazgo que podría sugerirnos la existencia de clones que pueden permanecer metabólicamente inactivos durante mayores periodos de tiempo y por lo tanto ser los responsables de las recaídas de la enfermedad porque permanezcan latentes aún durante el tratamiento (18) desde el punto de vista del Programa de Control, sería muy importante poder ratificar este hallazgo puesto que serviría para predecir cuales son los pacientes que se encuentran con mayor riesgo de presentar recaídas de la enfermedad por portar clones específicos, para lo cual, se hace necesario tener como rutina la genotipificación de las cepas de los pacientes desde que se diagnostica la enfermedad y así saber a cuales pacientes se les debe reforzar la vigilancia después del tratamiento.

Sin desconocer que el presente es un estudio de tipo descriptivo, subrayamos que este es el primer estudio que se realiza en Colombia caracterizando genotípicamente las cepas de Mycobacterium leprae que circulan o han circulado en nuestro medio. Si se compara con los reportes mundiales, el número de muestras incluido es grande. Igualmente, el número de variables analizadas en estas muestras también fue amplio y cubrió los tres aspectos clásicamente considerados importantes en la transmisión de la enfermedad. Por lo anterior y teniendo en cuenta que fueron escasas las agrupaciones exclusivas de variables en un genotipo, se evidencia que el poder conjunto de estos cuatro marcadores no es muy robusto y que para mejorarlo sería conveniente ampliar el panel a los 16 marcadores que actualmente han sido desarrollados.

A pesar de lo reportado por el grupo de Stewart Cole (11), respecto a la poca estabilidad de estos marcadores a través del tiempo, en nuestro estudio si se pudo observar que varios genotipos se presentaron durante el periodo de cinco años, en pacientes con tiempos de evolución de la enfermedad ampliamente diferentes en casos epidemiológicamente relacionados.

En este estudio se corrobora que como Colombia representa un país donde la lepra es endémica, los estudios de este tipo podrían ser útiles para el establecimiento y evaluación de las estrategias de control de la enfermedad, específicamente en lo que se refiere a la necesidad de implementar rutinariamente la genotipificación de las cepas, para identificar localizadamente la necesidad de incrementar la actividad de seguimiento de convivientes y la actividad de vigilancia de pacientes pos tratamiento que conjuntamente ayudarán a lograr la eliminación de la lepra, como problema de salud pública en nuestro medio 
Agradecimientos. Esta investigación fue totalmente financiada por el Centro Dermatológico Federico Lleras Acosta E.S.E., bajo el proyecto código 4000-16.2H. Los autores agradecen al doctor Patrick Brennan de la Universidad de Colorado (USA) por la donación de los ADN de referencia y de los oligonucleótidos iniciadores utilizados para la amplificación de los VNTRs. Igualmente agradecemos a AppliedMath's org, por el amable suministro (open access) del programa Gel Comprar II utilizado para análisis genotípico.

\section{REFERENCIAS}

1. Castiblanco M, Gómez J. Situación de la lepra en Colombia, 2006. Inf Quinc Epidemiol Nac 2007;12(18):273-288.

2. Scollard D, Adams L, Gillis J, Krahenbuh L, Truman R, Williams D. The continuing challenges of leprosy. Clin Microbiol Rev 2006;19(2):338-381.

3. Truman R, Fontes A, De Miranda AB, Suffys $P$, Gillis T. Genotypic variation and stability of for variable number tandem repeats an their suitability for discriminating strains of Mycobacterium leprae. J Clin Microbiol 2004;42(6):2558-2565.

4. Grothouse N, Rivoire B, KIM H, Brennan P, Vissa V. Multiple polymorphic loci for molecular typing of strains of Mycobacterium leprae. J Clin Microbiol 2004;42(4):1666-1672.

5. Matsuoka M, Zhang L, Budiawan T, Saeki K, Izumi S. Genotyping of Mycobacterium leprae on the basis of the polymorphism of TTC repeats for analysis of leprosy transmission. J Clin Microbiol 2004;42(2):741-745.

6. Supply P, Mazars E, Lesjean S, Vincent V, Gicquel B, Locht C. Variable human minisatellitelike regions en Mycobacterium tuberculosis genome. Mol Microbiol 2000;36:762-771.

7. Fontes A, Truman R, De Miranda A, Suffys P, and Gillis T. Poster presentation during " $16^{\text {th }}$ international Leprosy Congress", Agosto 4-9, Salvador, Brazil, 2002. Abstract book PM\&BM13, p.252.

8. Leproma. Paris: Instituto Pasteur [Internet] Disponible en: http://genolist.pasteur.fr/Leproma/ Acceso: 10 de diciembre de 2005.

9. Lg mb. [Internet].Paris: Instituto Pasteur. The Unit for Genetique Moleculaire Bacterienne. Disponible en: http://www.pasteur.fr/recherche/unites/Lgmb/ Acceso: 10 de diciembre de 2005.

10. The Sanger Centre [Internet].Cambridge, UK: Wellcome Trust Genome Campus. Disponible en: http://www.sanger.ac.uk/Projects/M_leprae/ Acceso: 10 de diciembre de 2005.

11. Monot M, Honore N, Baliere C, Ji B, Soe S, Brenen, P, Cole S. Are variable number tanden repeat (VNTR) Appropriate for genotyping Mycobacterium leprae? J Clin Microbiol 2008;46(7):2291-2297.

12. Fine P. Leprosy: the epidemiology of a slow bacterium. Epidemiological Rewiews 1982;4:161168.

13. Groh JL, Luo Q, Ballard JD, Krumholz LR. Genes that enhance the ecological fitness of Shewella oneidensis MR-1 in sediments reveal the value of antibiotic resistance. Appl Environ Microbiol 2007;73(2):492-498.

14. Casanova JL, Abel L. The human model: a genetic dissection of immunity to infection in natural conditions. Nat Rev Immunol 2004;4:55-66.

15. Siddiqui MR, Meisner S, Tosh K, Balakrishnan K, Ghei S, Fisher SE, et al. A major susceptibility locus for leprosy in India maps to chromosome 10p13. Nat Genet 2001;27:439-441.

16. Miller EN, Jamieson SE, Joberty C, Fakiola M, Hudson D, Peacock CS, et al. Genome-wide scans for leprosy and tuberculosis susceptibility genes in Brazilians. Genes Immun 2004;5:6367.

17. Wickham ME, Brown NF, Boyle EC, Coombes BK, Finlay BB. Virulence is positively selected by transmission success between mammalian hosts. Curr Biol 2007;17(9):783-788.

18. Toman K. Case detection, treatment, and monitoring-questions and answers In: Tuberculosis. Second edition Hong Kong Edited by T. Frieden, 2004 WHO/HTM/TB/2004.334. 